This is the accepted version of the following article: Guo, Wusheng et al. "Water-soluble Au nanoparticles : from catalytic selective nitroarene reduction in water to refractive index sensing" in Chemistry, an Asian journal, vol. 10 issue 11 (Nov 2015), p. 2437-2443, which has been published in final form at 10.1002/asia.201500290. This article may be used for non-commercial purposes in accordance with Wiley Terms and Conditions for Self-Archiving.

\title{
Water-soluble Au nanoparticles: from catalytic selective nitroarene reduction in water to refractive index sensing
}

\author{
ChemSusChem Pleixats ${ }^{\text {ta] }}$ and Alexandr Shafir ${ }^{\text {[al }}[$ [b]
}

\begin{abstract}
FUl Papers Abstract: Water-soluble gold nanoparticles (Au NPs) stabilized by a nitrogen-rich PEG-tagged substrate have been prepared by reduction of $\mathrm{HAuCl}_{4}$ with $\mathrm{NaBH}_{4}$ in water at room temperature. The morphology and size of the nanoparticles can be controlled by simply varying the gold/stabilizer ratio. The nanoparticles have been fully characterized by TEM, HRTEM, ED, EDS, UV-vis, $p$-XRD and elemental analysis. The material is efficient as a recyclable catalyst for the selective reduction of nitroarenes with $\mathrm{NaBH}_{4}$ to yield the corresponding anilines in water at room temperature. Furthermore, the potential ability of the Au NPs as a refractive index sensor due to their localized surface plasmon resonance (LSPR) effect has also been assessed.
\end{abstract}

\section{Introduction}

Within the ever-growing field of the application of metal nanomaterials, the scientific community has dedicated a large amount of effort to studying the properties and potential applications of gold nanoparticles (Au NPs). This rising interest is due, in large part, to the remarkable Au NPs stability, biocompatibility, as well as their size-related electronic and optical properties (quantum-size effect). Thus, Au NPs have found applications in areas as diverse as chemical and biological sensing ${ }^{[1,2]}$ catalysis, ${ }^{[3]}$ and biomedicine. ${ }^{[4]}$ Various methods have been developed for the preparation of gold NPs, including both the "top-down" (mainly physical manipulation) and "bottom-up" procedures, ${ }^{[5,6]}$ the latter consisting mainly of chemical methods, with the reduction of gold salts being the most common. For the use in catalytic applications, it is often desirable to obtain small-size Au NPs, thus maximizing th fraction of gold atoms on the surface, given that these atoms a generally responsible for the catalytic activity. Unfortuna metal NPs in solution are subjected to facile agglomeration towards the thermodynamically favored bulk metal, with the consequent loss of their catalytic activities. The most popular strategies for chemists to overcome this problem consists in supporting Au NPs on an inorganic matrix ${ }^{[5]}$ or preventing the aggregation of soluble Au NPs with effective stabj -agents, ${ }^{[7]}$ including those based on polymers. ${ }^{[8]}$ The rran the protecting agent, in turn, determines the solubility prope the metal nanoparticles. Thus, a suitable chgice of the stabiliz may render the nanomaterial soluble in medium and may even facilitate the recy

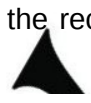

[a] Dr. W. Guo, Prof. R. Pleixats, Dr. A. Shafir Department of Chemistry and Centro de Innovación en Química Avanzada (CINQA)

Universitat Autònoma de Barcelona

08193-Cerdanyola del Vallès, Barcelona, Spain

E-mail: roser.pleixats@uab.cat; ashafir@iciq.es

[b] Dr. A. Shafir

Institute of Chemical Research of Catalonia (ICIQ)

Av. Països Catalans 16, 43007-Tarragona, Spain

Supporting information for this article is given via a link at the end of the document.
NP solubility in aqueous media is highly attractive, given that water is a low cost environmentally benign madium, non-toxic and safe, which is of particular interest not only an laboratory but also for industrial processes. However, may the stabilized Au NPs reported in the literature are not w which, for a catalytic application, greatly vecrease re chances of the substrate to contact the catalyst ous medium, resulting in long reaction times or low activities

Besides its effect on solubility, ture and pon the stabilizer employed is also a to control he size of the NPs formed, which, as already entioned, is of the key to a high catalytic activity. In additio the size of a NP o translates into its electronic properties, $\mathrm{ch}$, for Au NPs ncludes the Localized Surface Plasmo Resc (LSPR) rects, making them excellent scaffolds in rious orications. ${ }^{[1,2]}$ For instance, recent reports sho the potential of Au NPs as sensors of heavy metal ions in ueous solutions. ${ }^{[10]}$ Thus, to prepare water-solub und is or significance, especially when considering therr canum in aqueous medium, as well as their sensing and biologicarapplications.

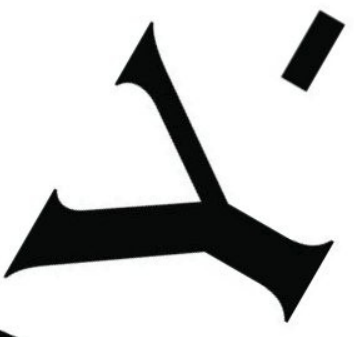

As a develop, ent of protocols to prepare Au NPs that are stable and easily dispersible in water. The most common method is th growth of Au NPs in the presence of hydrophilic thiols cor aining short ethylene oxide oligomers or long (ethylene glycol) (PEG) chains. ${ }^{[13,14]}$

Our group previously described ${ }^{[15]}$ water-soluble Pd NPs, stabilized by two different star-shaped PEG-tagged substrates, as recyclable catalysts for cross-coupling reactions. However, these ligands fell short for the formation of other metal nanocatalysts, including $\mathrm{Au}$ and $\mathrm{Rh}$, in terms of yields in the nanoparticles synthesis and/or reproducibility of the procedures. This led us to design a new nitrogen-rich polyoxyethylenated stabilizer $\mathbf{L}$ with a triazine core and triazole moieties in the branches (Scheme 1). Thus, we recently reported ${ }^{[16]}$ the synthesis of this PEG-tagged substrate $\mathbf{L}$ via copper-catalysed alkyne-azide cycloaddition (CuAAC, click chemistry), and the use of this molecule as stabilizer for the formation of size and morphology controllable Rh NPs. The materials proved to be excellent recyclable catalysts in the hydrosilylation of internal alkynes (Scheme 1). 

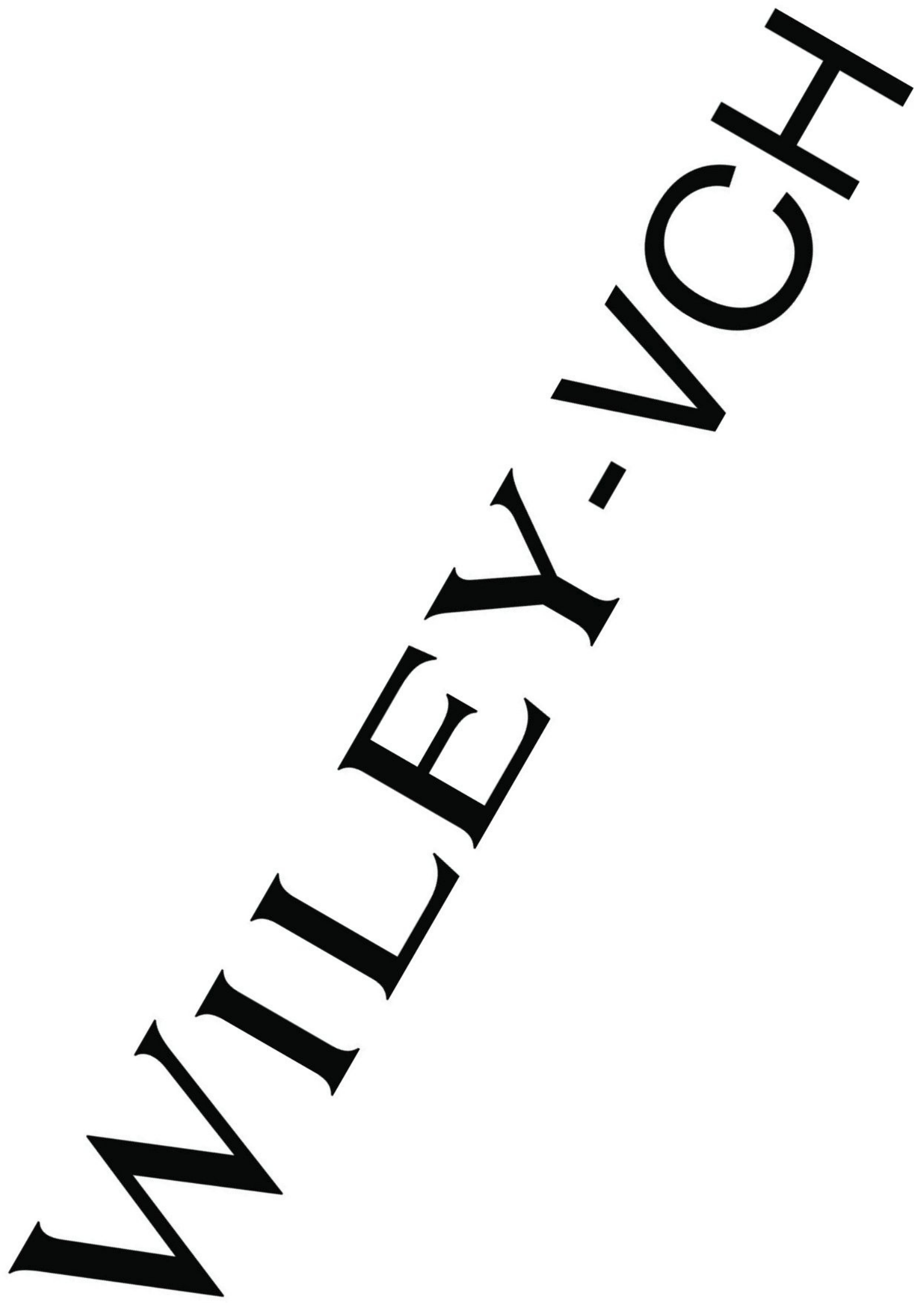
Table 1. Preparation of Au NPs stabilized by $L$ from the reduetion of tetrachloroauric acid with sodium borohydridela

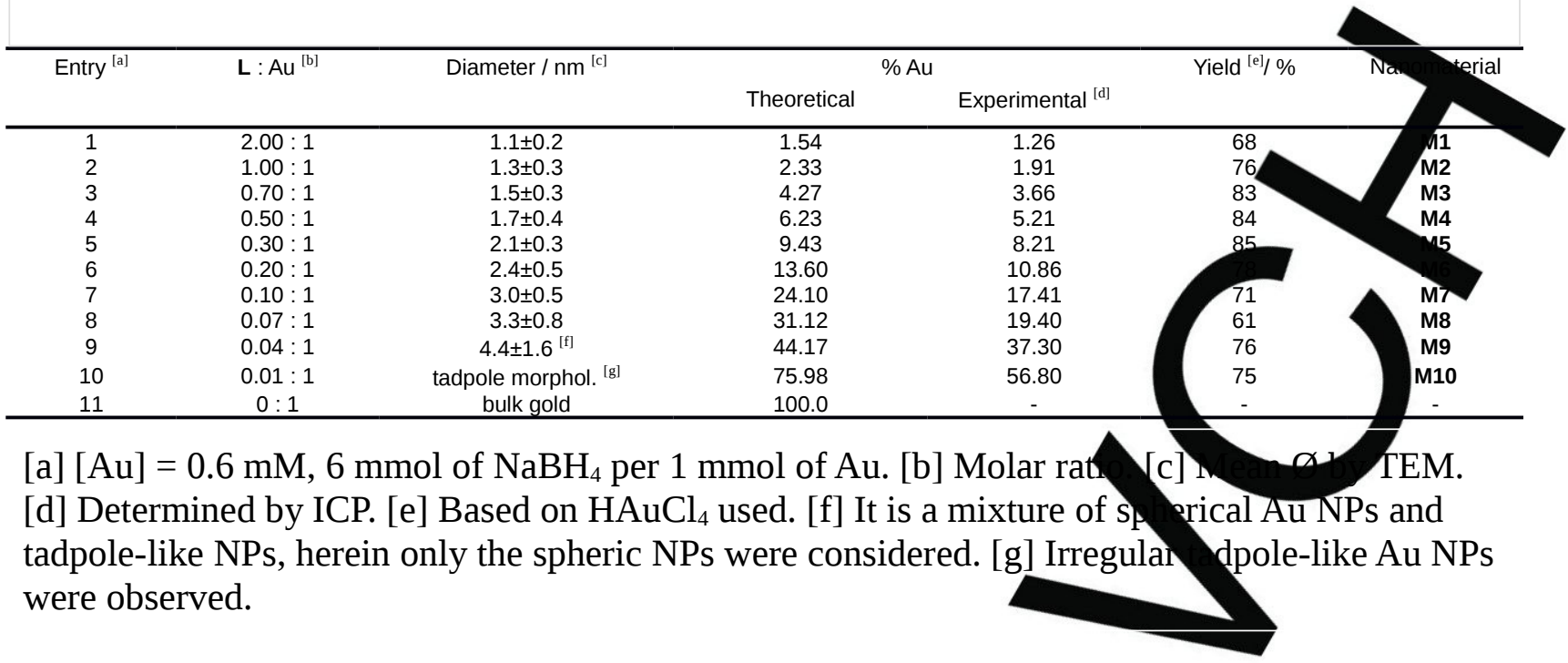

This three-fold symmetric molecular architecture was initially proposed taking into account the potential benefits of both the nitrogen-rich structure and the PEG moieties on the nanoparticle stabilization and also the operational convenience of bonding via triazole linkages built by a click reaction. ${ }^{[17,18]}$ Encouraged by these results with Rh NPs, herein we report our latest results on using the stabilizer $\mathbf{L}$ for the efficient preparation of size and morphology controllable Au NPs, and their subsequent use as recyclable catalysts in the selective nitroarene reduction in water.

\section{Results and Discussion}

\section{Preparation and characterization of Au NPs}

A chemical reduction method was applied to test the stabilizing ability of $\mathbf{L}$ for the formation of Au NPs, whereby $\mathrm{HAuCl} \cdot 3 \cdot 3 \mathrm{H}_{2} \mathrm{O}$ was used as the source of gold and $\mathrm{NaBH}_{4}$ as a reducing reagent, with reactions run in wa at room temperature.

We initially examined the formation of Au NPs with L/Au ratio of $=2.00 / 1$ (Table 1 , entry 1 ). It was found that, upo addition of an excess of the $\mathrm{NaBH}_{4}$ solution $5 \mathrm{mmol}$ per mmol $\mathrm{Au})$, the reaction mixture immediately ont a color change from light yellow to black, b with no pre bulk metal after stirring overnight. The ction mixture filtered through a Millipore filter and th filtrate was extro ted with dichloromethane. In this manner, $M$. was obtained as a black solid upon removal of solvent. The ${ }^{1}$ VMR spectrum of M1 was identical to that on supporting information). TEM analysis con of spherical and well dispersed nanoparticles vith a mean size of $1.1 \mathrm{~nm}$ (based on 150 particles measured, able 1, entry 1). The yield with respect to the $f$ as as $68 \%$ on the basis of the material's $1.26 \% \mathrm{Au}$ ). Although the size of the NPs obtained appeared adequate for catalytic applications we were in rested in probing the influence of the stabilizer-to- tal ratio on the nanoparticle size, morphology and reactivity Indeed, the size and morphology of metal nanomaterials ve been reported to play a key role in the activ ${ }^{1}$ ivity for some catalytic systems. ${ }^{[19]}$ Thus a car $/ A u$ ratio under the present reaction conditions was perform (Table 1, entries 1-10).

As summarized in Table 1 (entries 1-8), although spherical well dispersed nanoparticles (M1-M8) were formed over a wide L/Auratio, as the L/Au ratio was gradually decreased from 2.00 to $0.07 /$ he particle size rose from $1.1 \pm 0.2$ to $3.3 \pm 0.8$ $\mathrm{nm}$ in a ma er somewhat analogous to what had been served wit Rh NPs. ${ }^{[16]}$

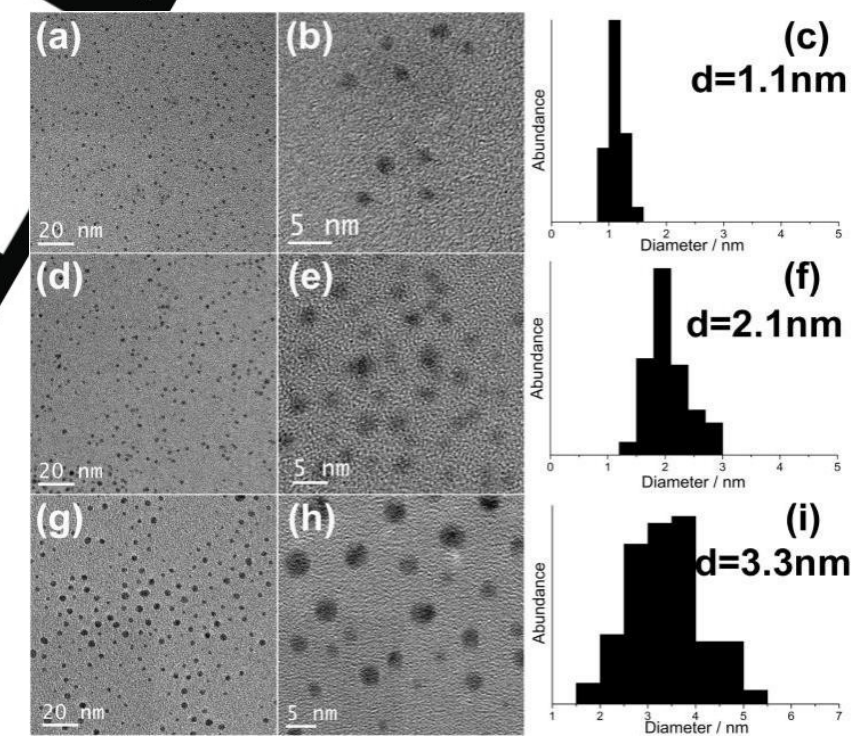


Figure 1. TEM and HRTEM images, and the corresponding size distributions of the materials M1 (a-c), M5 (d-f) and M8 (g-i).

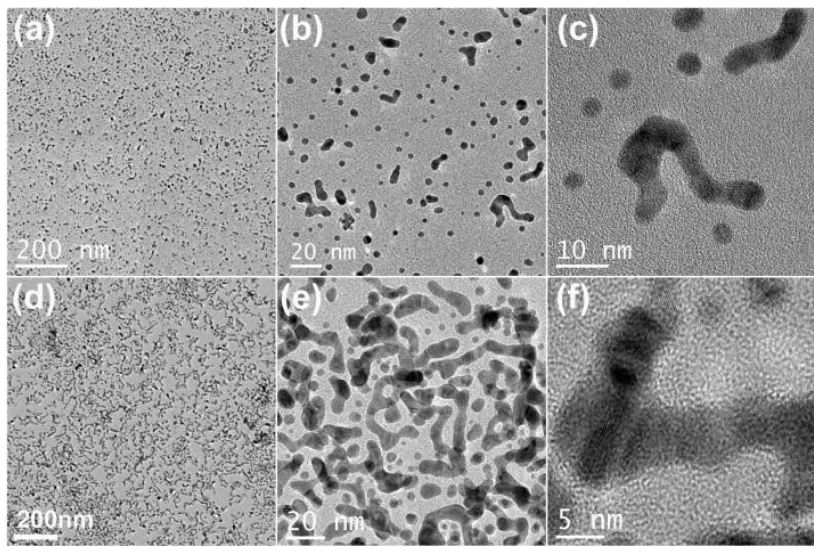

Figure 2. TEM images under different magnifications and the corresponding HRTEM images: M9 (a-c) and M10 (d-f).

Selected TEM and HRTEM images, and the corresponding size distributions of the materials $\mathbf{M 1}, \mathbf{M} 5$ and $\mathbf{M 8}$ are shown in Figure 1. Since the size of the Au NPs (and of the metal nanoparticles, in general) should, in principle, be determined by the relative rates of nucleation and particle growth, the formation of smaller nanoparticles with a higher amount of stabilizer can be ascribed to the more efficient suppression of the nanoparticle growth at higher stabilizer-to-metal ratios. thoroughly, the materials were found to be completely soluble in water as depicted in Figure 3a.

Further structural investigation was conducted by registering the UV-vis spectra of the materials M1-M10 in THF (Figure 3b). For particles larger than $2 \mathrm{~nm}$ (M5-M10), the appearance of a broad shoulder at 450-600 nm could be discerned representing the localized surface plasmon resonance (LSPR). This absorption shows an appreciable red shift with the decreasing L/Au ratio $(0.30 / 1$ to $0.01 / 1)$, consistent whe known ${ }^{[20]}$ dependence between the LSPR band and the ou size. Nevertheless, for materials M1-M4 containing partic than $2 \mathrm{~nm}$, the LSPR band was not clearly obser d, in gou agreement with previous research. ${ }^{[11]}$

The $p$-XRD patterns (Figure $3 c$ ) of M5, in a $\mathbf{M} 7$ showed two intense peaks located in the ranon- $2 \theta=1$ ottributed to the crystalline (120) and (117 pranes of the PEQ lagged stabilizer. ${ }^{[16,21]}$ Four additional eak diffractio ands in the range $2 \theta=40^{\circ}$ to $80^{\circ}$ were as gned to the (111 (200), (220) and (311) planes of fcc gold it ce, respectively These data fit well with the ED analysis. he Energy-Di ersive X-ray Spectroscopy (EDS) spe $m$ of supporting information as a represe tive exampre, confirming the presence of gold.

\section{$\begin{aligned} & \text { Reduction of nitroa } \\ & \text { nanoparticles }\end{aligned}$}

Among the many potential catalytic applications of gold nanoparticle ${ }^{[3]}$ the red tion of nitroarenes in water was chosen as model $\mathrm{p}$ cess. This reaction has been widely studied, not o ly because the corresponding anilines are very important ind trial raw materials, but also because the nitroaryl compd nds are common organic pollutants in indy wastewaters due to their use as butung olocks in areas such as dyes, pesticides, explosives and as orgâric solvents. Sodium borohydride is one the most employed reducing agents for nitroarenes under metal nanoparticles catalysis. ${ }^{[23,24,25]}$ However, in many cases the $r$ ctivity s vdies have been limited to the model reduction of nitrophen to $p$-nitroaniline. We were interested in br dening scope of this reaction by performing the ective re ction of the nitro group even in the presence of ans ve functional groups under mild reaction conditions in aqu smedium.

A first reaction was carried out with nitrobenzene as model con hound (at $0.5 \mathrm{~mol} \%$ of Au loading using M5) employing 50 $\mathrm{mr}$ of $\mathrm{NaBH}_{4}$ per mmol of substrate ${ }^{[23]}$ (Table 3, entry 1). To delight, monitoring the reaction by TLC showed a spot-toot conversion of the starting material to a new product after Just $1 \mathrm{~h}$ at room temperature. Taking into account that the stabilized Au NPs are soluble in water but not in diethyl ether, the reaction mixture was extracted with diethyl ether, affording the pure aniline in a quantitative yield after solvent removal. Lowering the amount of the sodium borohydride to 35 $\mathrm{mmol} / \mathrm{mmol}$ of nitrobenzene (Table 3 , entry 2 ), still allowed for an efficient, although somewhat slower, reaction.

Keeping the amount of sodium borohydride constant at a $35: 1$ molar ratio, the reaction time did not change significantly on lowering the loading of catalyst to $0.3 \mathrm{~mol} \%$ (Table 3 , entry 3 ), although at this catalyst loading an attempt to further decrease the $\mathrm{NaBH}_{4}$ equivalents led to a lower product yield and a considerable more sluggish reaction (Table 3 , entry 4 ). As a reference experiment, no conversion took place in the absence of the Au catalyst (Table 3, entry 5). Similarly, no 
reaction occurred in the absence of sodium borohydride (Table

3 , entry 6). It is worth noting that all the reactions mentioned above were performed without any special precaution in water under air at room temperature.

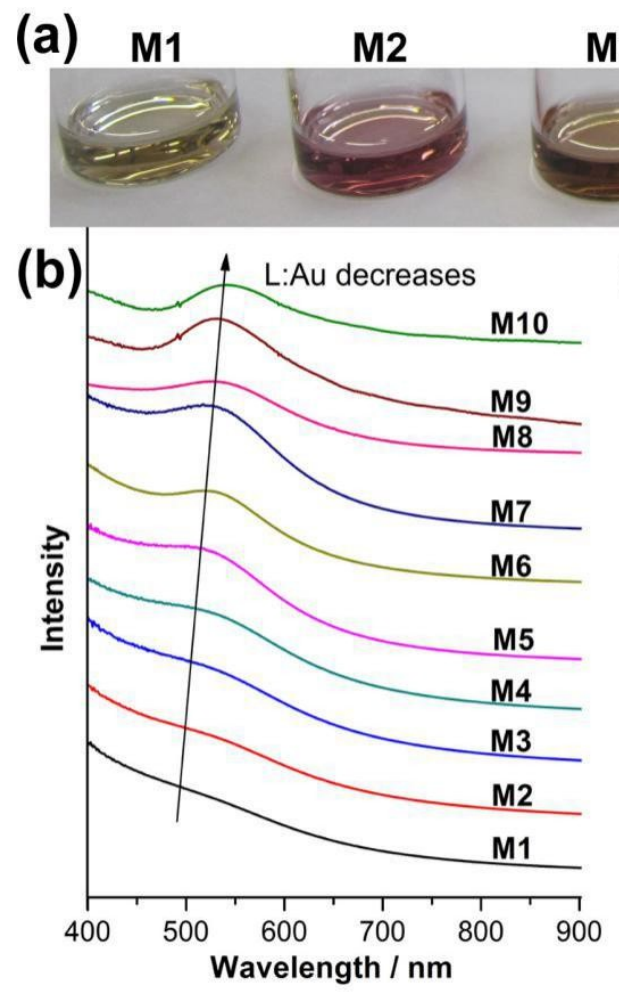

M3

M4

M5

M7
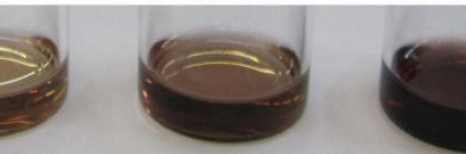

(c)
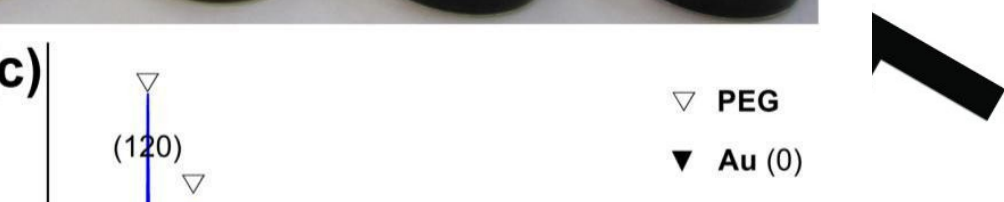

$(111)$

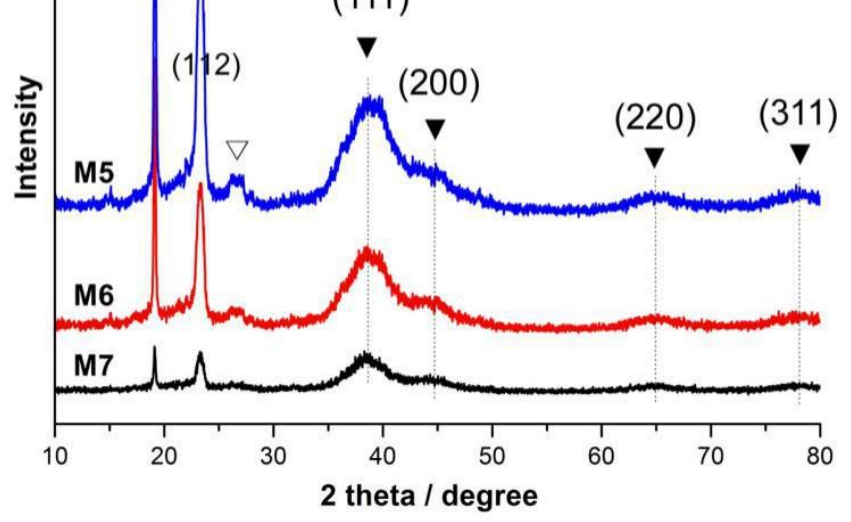

Figure 3. (a) Optical photographs of Au NPs (2 mg) in $\mathrm{H}_{2} \mathrm{O}(1 \mathrm{~mL})$; (b) Absorption spectra of Au NPs in $(0.2 \mathrm{mM}$ of Au); (c) p-XRD patterns of select. Au NPs.

Table 3. Optimization of the reaction conditions ${ }^{[a]}$

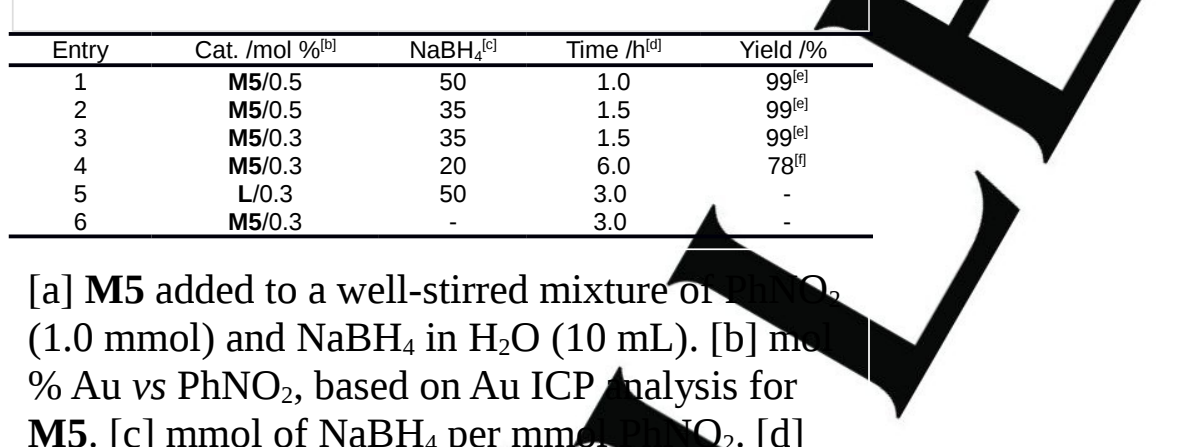

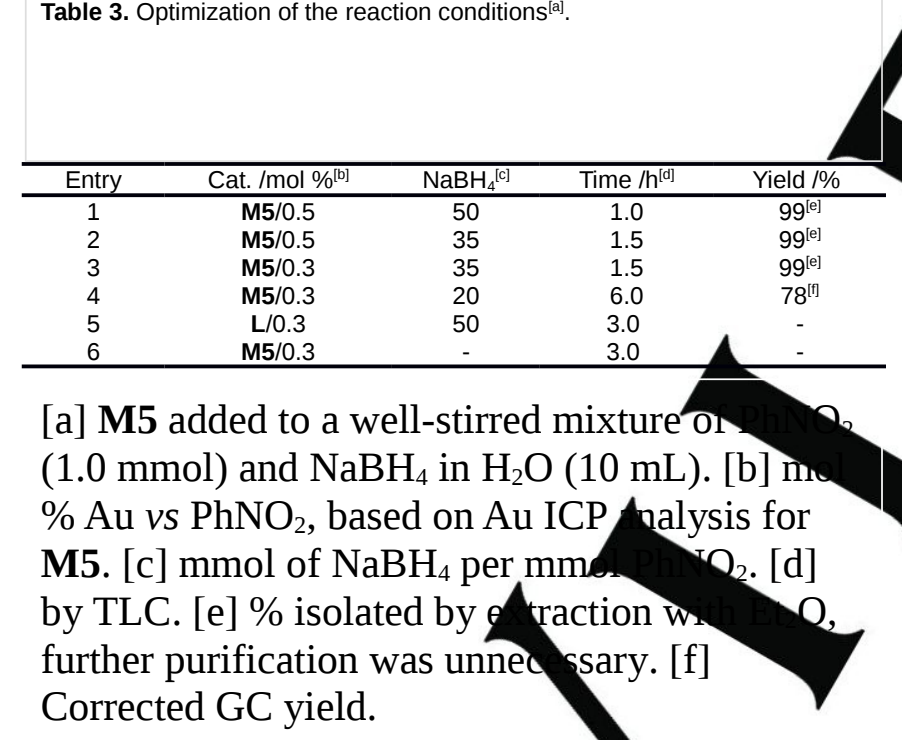

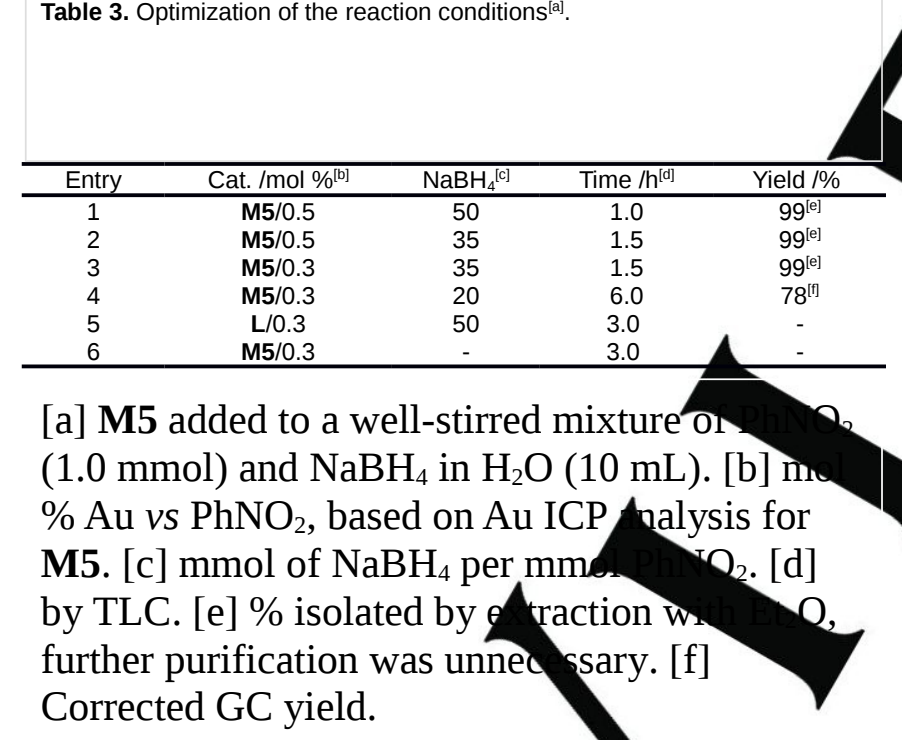

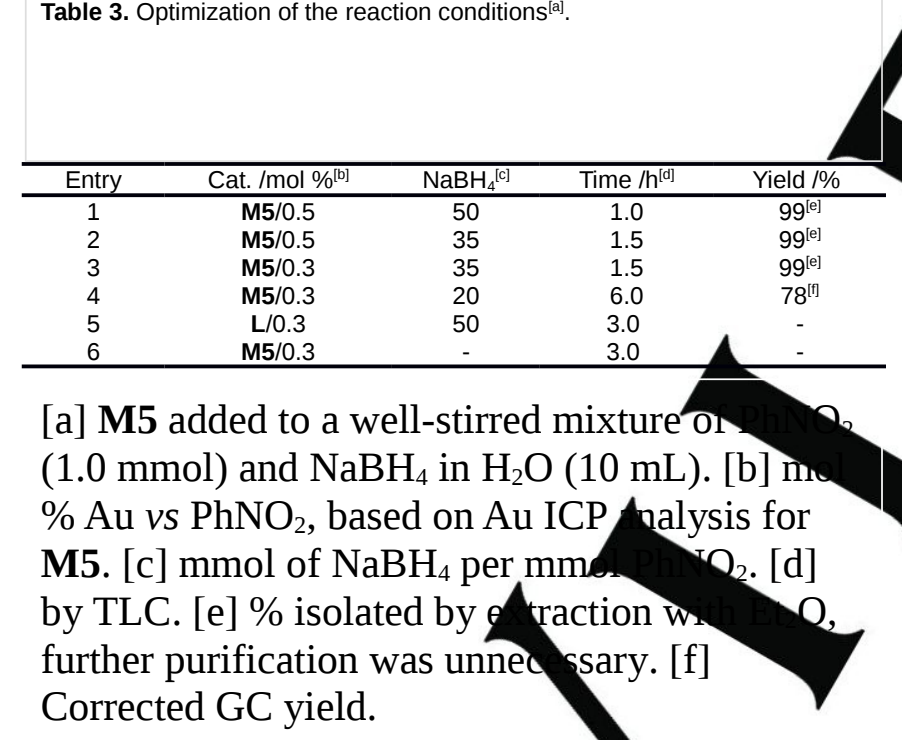

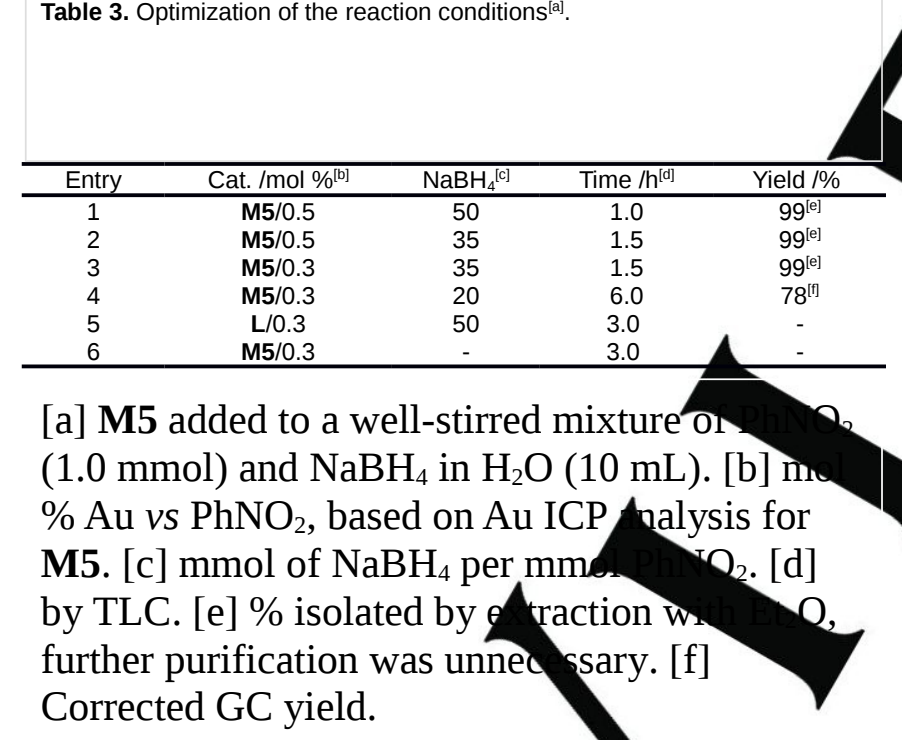
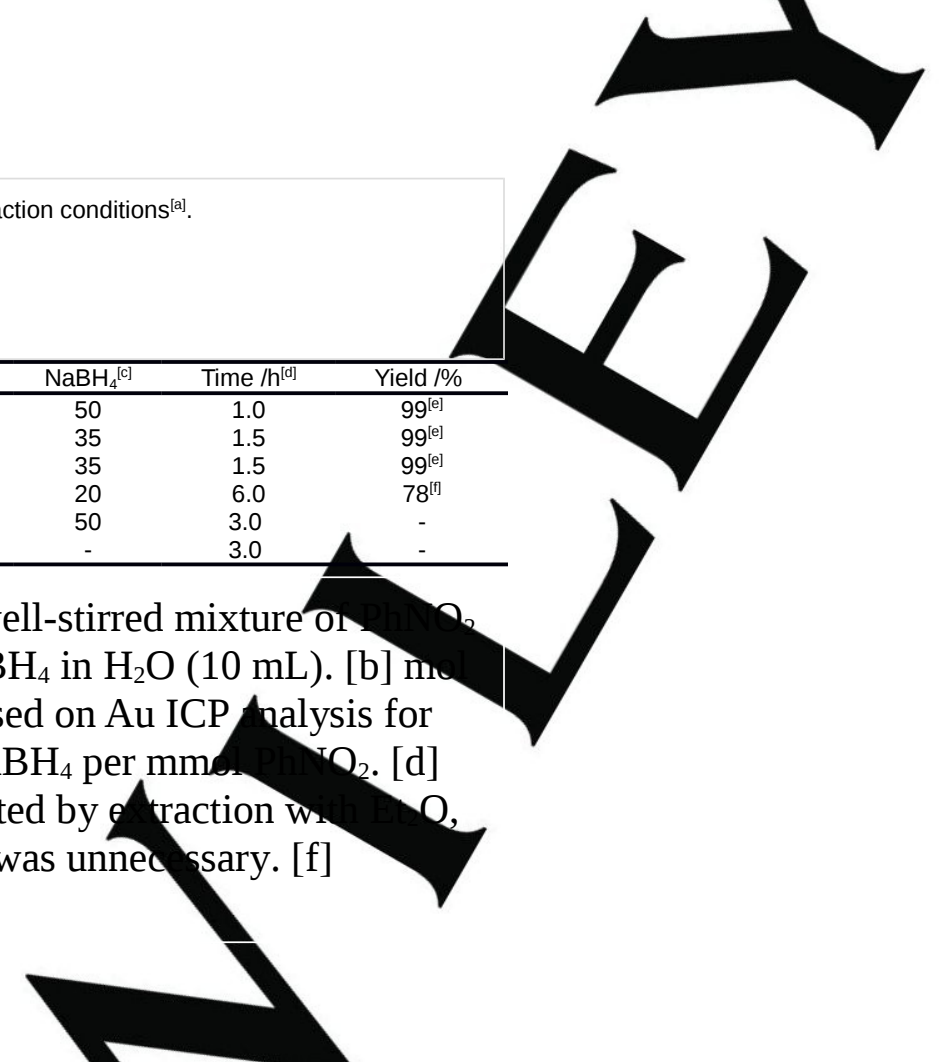

Under the optimized conditio $\mathbf{s}(0.3 \mathrm{~mol} \% \mathrm{Au}, 35 \mathrm{mmol}$ of sodium borohydride/mmol of substrate), we first investigated the reusability of $\mathbf{M} 5$ in the reduction ol itrobenzene, taking advantage of its solubility properties (soluble in water, insoluble in diethyl ether). Thus, once t aqueous phase reary 
activity, probably due to some loss of catalyst, but giving a respectable isolated yield of $78 \%$ in the fourth run. ${ }^{\left[{ }^{[6]}\right.}$ The reaction scope was then extended to several additional nitroarenes using M5 as a representative catalyst (Table 4). The protocol was found to be tolerant of the presence of electron-withdrawing and electron-donating substituents on the aromatic ring. Thus, the catalyst was very effective for the reduction of 3-nitroaniline and 4-nitrobenzenesulfonamide to the corresponding products in quantitative yields in less than an hour (Table 4, entries 5-6). The $p$-nitroanisole gave an excellent yield of $p$-methoxyaniline albeit at a longer reaction time (Table 4 , entry 7). The reduction of $p$-bromonitrobenzene furnished $p$-bromoaniline in $72 \%$ isolated yield with only trace of dehalogenated product detected (Table 4, entry 8). The catalytic system was also able to reduce the $p$-nitrobenzonitrile and methyl $p$ nitrobenzoate, yielding the corresponding anilines in $89 \%$ and $82 \%$ isolated yields, respectively (Table 4 , entries $9-10$ ); the $-\mathrm{CN}$ and -COOMe remained unaltered under the reaction conditions.

Last, the catalytic activity of M5 was tested on p-nitrophenol, an environmentally hazardous substance included $\mathbf{s}, \mathrm{U}$. S. Environmental Protection Agency on its "Priority Pollutant List". ${ }^{[24]}$ Remarkably, the reduction of $p$-nitrophenol was complet min affording $p$-aminophenol in quantitative yield (Table 4 , entry 11). The faster reaction with this substrate comp reduction of $p$-methoxyaniline (compare entries 7 and 11) must be due to its higher hydrophilicity derived hydroxyl group. This reaction was also used as a model to investigate the catalytic activities of other Au NPs Sys (M1 and M9) featuring nanoparticle sizes and morphologies different from those of M5 (Table 4, entries 12-13). As exmed, M9 red lower activity when compared with M5, which should be ascribed to the higher particle size and, consequent ower number of ac sites on the surface. However, the catalysis using $\mathbf{M} 1$ needed the longest reaction time (22 min) which mid be rationaliz y the excess of stabilizer blocking the particle surface and causing a drop in catalytic activity.

Table 4. Reduction of nitroarenes catalyzed by Au NPs ${ }^{[a]}$.

\begin{tabular}{|c|c|c|c|c|c|}
\hline Entry & Product & Cat. & $\begin{array}{c}\text { Conv. } \\
1 \%\end{array}$ & Time $^{[\mathrm{b}]}$ & $\begin{array}{l}\text { Yield } \\
1 \% \%^{[c]} \\
\end{array}$ \\
\hline 1 & & M5 & $>99$ & $1 \mathrm{~h}$ & $99^{[d]}$ \\
\hline 2 & & M5 & $>99$ & $1.5 \mathrm{~h}$ & $88^{[\mathrm{e}]}$ \\
\hline 3 & & M5 & 94 & $2 \mathrm{~h}$ & $86^{[f]}$ \\
\hline 4 & & M5 & 87 & $5 \mathrm{~h}$ & $78^{[g]}$ \\
\hline 5 & & M5 & $>99$ & $35 \mathrm{~min}$ & 99 \\
\hline 6 & & M5 & $>99$ & $48 \mathrm{~min}$ & 99 \\
\hline 7 & & M5 & $>99$ & $3 \mathrm{~h}$ & 98 \\
\hline 8 & & M5 & $>99$ & & \\
\hline 9 & & M5 & & $1.5 \mathrm{~h}$ & \\
\hline 10 & & IVI & & & \\
\hline 11 & & M5 & & $11 \mathrm{~min}$ & $9^{[\mathrm{h}]}$ \\
\hline 12 & & M9 & & $16 \mathrm{~min}$ & 99 \\
\hline 13 & & M1 & & $22 \min$ & 99 \\
\hline
\end{tabular}

[a] To a well stirred mix mmol) and $\mathrm{NaBH}_{4}(35 \mathrm{~mm} \mathrm{v})$ in water $(10 \mathrm{~mL})$ at room tempera"ure was add the catalyst $(0.3$ mol\%). [b] TL or connonis pg. [c] Isolated 
yield. [d-g] Consecutive cycles with the same batch of catalyst. [h] TON (mol product/mol catalyst $)=330$, TOF $($ TON/time $)=30 \mathrm{~min}^{-1}$.

It is worth noting that the scope of the present catalytic system is broader than in most of other reports, where only wophenol was used to test to catalytic activity. In this context, an interesting comparison can be made with a recent report from $\mathrm{Kantam}$ laboratory, describing a supported gold nanocatalyst for the reduction of a wide range of nitroarenes in water, ${ }^{[23]}$ and $p$ catalytic TON's of up to 207 when employing a 50-fold excess of the reducing agent. As seen in Table 1, our new A reaches a TON of $330\left(\right.$ TOF $=30$ min-1) while requiring less $\mathrm{NaBH}_{4}$ loading.

\section{Evaluation of the gold nanoparticles as refractive index sensor}

One of the simplest applications of the nanoparticle LSPR phenomenon is the detection of changes their environment through shifts in the LSPR peak wavelength. ${ }^{[1]}$ In order to investigate the ability sensors, M7 (mean size of $3.0 \pm 0.5 \mathrm{~nm}$ ) was chosen as representative nanomaterial. The n concentration of $0.2 \mathrm{mmol} / \mathrm{L}$ ) in a series of solvents covering a range of refractive index values. $\mathrm{S}$ conducted in carbon disulfide $(n=1.627)$, toluene $(n=1.496)$, chloroform $(n=1.447), t$-butanol

S cifica
1.627), toluene $(n=1.496)$, chloroform $(n=1.447)$, $t$-butanol $(n=1,9)$ and water $=1.333)$.
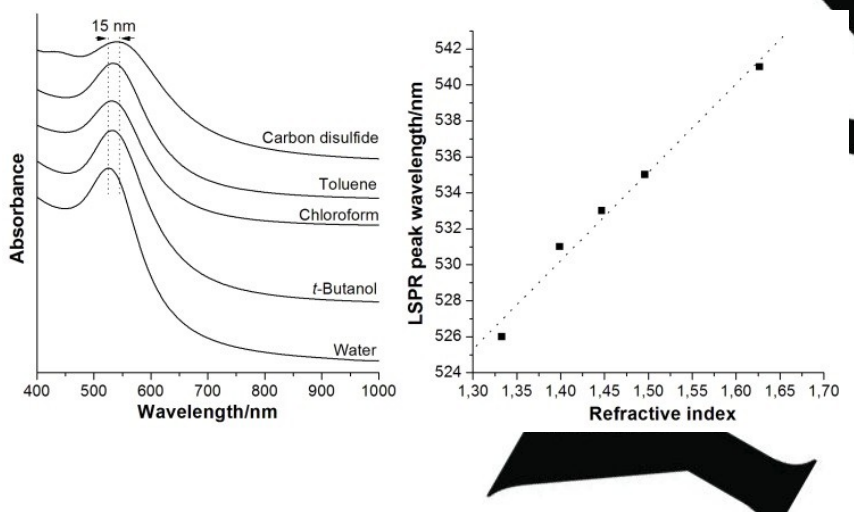

Figure 4. Optical absorbance of $\mathbf{M 7}$ with gold concentration of $0.2 \mathrm{mM}$ in carbon disulfide, toluene, chlorofoln, $t$-butanol and water (a); plotting of the peak wavelength versus refractive index indicating the linear correlation (b).

The absorbance spectra of these five samples (Figure 4a) sh maximum absorbance of the samples experienced a notable indeed, be used as refractive index sensors. As can be seent LSPR peak wavelength and the medium refractive index, in good ag

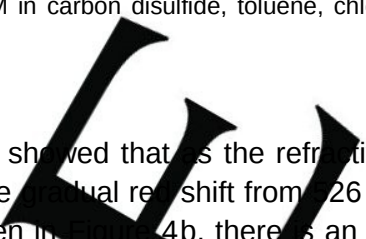

tive index increased from 1.333 to 1.627 , the $26 \mathrm{~nm}$ to $541 \mathrm{~nm}$, indicating that Au NPs could, an approximately linear correlation between the $4 \mathrm{~b}$, the he reported theory. ${ }^{[1,2,27]}$

\section{Conclusions}

A nitrogen-rich polyoxyethylenated substrate was tetrachloroaurate(III) in water at room temperature. The the gold-to-stabilizer ratio. The newly prepared nanomateria and elemental analysis. Further investigatis showed that the

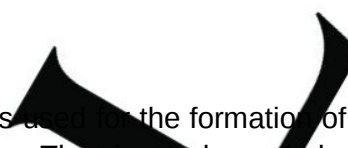
ff water soluble Au NPs upon $\mathrm{NaBH}_{4}$ reduction of hydrogen logy of the nanoparticles can be controlled by simply varying NPs displayed excellent activity as catalyst for the mild and selective reduction of differently substitute renes to the corresponding anilines using $\mathrm{NaBH}_{4}$ as reducing agent in water at room temperature. The new nanoc alyst coulan the recycled (four runs) taking advantage of its solubility properties. Thus, the product was directly extracted frorr reaction mean diethyl ether, leaving the Au NPs-containing water phase ready for the next run. The Au NPs were also succes wlly tested as retry ive index sensors.

\section{Experimental Section}

The stabilizer $\mathbf{L}$ was prepared accor $g$ to our previous teport. ${ }^{[16]}$ All NMR spectra were recorded with Bruker Avance360 (360 MHz for $\left.{ }^{1} \mathrm{H}\right)$ and Avancell $400 \mathrm{MHz}\left(400 \mathrm{MHz}\right.$ for $\left.{ }^{1} \mathrm{H}\right) \mathrm{S}$, ctrometers. ICP measurements of gold content were carried out at the Serveis Cientifico-Tècnics of the Universitat de Barcelon with a multichann Perkin-Elmer instrument, model Optima 3200 RL. TEM and ED analyses were performed at the Servei de Microscòpia of the $U$ vrcelona, with a JEOL JEM-2010 model instrument operating at $200 \mathrm{kV}$. The nanoparticle sizes were determined by measuring 150 parnumal Micrograph software (Gatan, Inc.) and were subsequently averaged to produce the mean NP 
diameter. UV-vis spectra were acquired at room temperature using a HP8453 spectrophotometer (Agilent) running a ChemStation software. The powder X-ray diffraction ( $p$-XRD) patterns were collected using a conventional powder X-ray diffractometer (Siemens D5000) at the Institut de Ciència dels Materials de Barcelona.

General procedure for the preparation of Au NPs (M5 as an example): To a solution of the stabilizer $\mathbf{L}$ (112 mg, 0.018 mmol) and hydrogen chloroaurate(III) trihydrate $(24 \mathrm{mg}, 0.06 \mathrm{mmol})$ in water $(100 \mathrm{~mL})$ under nitrogen atmosphere, was added dropwise via a syringe (over 2 minutes) a solution of $0.1 \mathrm{M}$ sodium borohydride $\left(3.6 \mathrm{~mL}, 0.36 \mathrm{mmol}\right.$ of $\left.\mathrm{NaBH}_{4}\right)$. The mixture was stirred at room temperature overnight, and then it was filtered through a Milli-Pore filter. The filtrate was extracted with dichloromethane $(6 \times 20 \mathrm{~mL})$ and the organic phase was dried over anhydrous sodium sulfate. After the removal of the solvent, Au NPs were obtained as a black powder (122 mg, Au \% $=8.21 \%, 85 \%$ yield with respect to the Au sed).

General procedure for the reduction of nitroarenes (reduction of $p$-nitrophenol as example): To a well stirred mixture of $p$-nitrophenoly 1.0 $\mathrm{mmol}$ ) and sodium borohydride $(1.324 \mathrm{~g}, 35 \mathrm{mmol})$ in water $(10 \mathrm{~mL}), \mathbf{M} 5$ was added $(0.3 \mathrm{~mol} \%$ Au catalyst loading, $0.007 \mathrm{~g})$ and the stirred at room temperature. Upon the completion of the reaction (TLC monitoring), the mixture was extracted with diethyl ether. The was dried over anhydrous $\mathrm{Na}_{2} \mathrm{SO}_{4}$ and then the solvent was evaporated under reduced pressure, affording pure $p$-aminop $99 \%$ yield).

\section{Acknowledgements}

Financial support from Ministerio de Ciencia e Innovación (MICINN) of Spain (Projects CTC 009-07881, CTC 011-22649), Consolider Ingenio 2010 (CSD2007-00006), Ministerio de Economía y Competitividad (MINECO) Spain (Proj /s CTQ201346705-R; CTQ2014-51912-REDC), a Ramón y Cajal scholarship to A. S. (MEC, Spain, RYC 6-00. Generalitat de Catalunya (SGR2009-1441; SGR2014-1105, SGR2014-1192) and China Scholarship Counch SC) Schorarship to W. G. are gratefully acknowledged.

Keywords: Water-soluble Au NPs • Reduction • Nitroarenes • Anilines

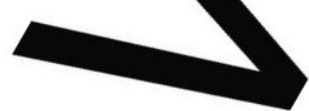

[1] K. M. Mayer and J. H. Hafner, Chem. Rev., 2011, 111, 3828-3857

[2] K. Saha, S. S. Agasti, C. Kim, X. Li and V. M. Rotello, Chem. Rev., 2012, 112, 2739-2779.

[3] a) A. Corma and H. García, Chem. Soc. Rev., 2008, 37, 2096-2126; b) M. Stratakis anćn, García, Chom. Rev., 2012, 112, 4469-4506; c) X. Zhang and Y. Ding, Catal. Sci. Technol., 2013, 3, 2862-2868; d) Y. Mikami, A. Dhakshinamoorthy, M Alvaro and H. García, Catal. Sci. Technol., 2013, 3, 5869; e) B. S. Takale, M. Bao and Y. Yamamoto, Org. Biomol. Chem., 2014, 12, 2005-2027.

[4] a) E. C. Dreaden, M. A. Mackey, X. Huang, B. Kang and M. A. El-Sayed, Chem. and M. V. Russo, J. Mater. Chem. B, 2014, 2, 4204-4220.

[5] J. M. Campelo, D. Luna, R. Luque, J. M. Marinas and A. A. Romero, ChemSusChem, 2009, 2, 18-45.

[6] a) M. C. Daniel and D. Astruc, Chem. Rev., 2004, 104, 293-346; b) P. Zha Ly. Li and D. Astruc, Coord. Chem. Rev., 2013, 257, 638-665; c) C. D. Pina, E. Falletta and M. Rossi, Chem. Soc. Rev., 2012, 41, 350-369.

[7] A. Roucoux, J. Schulz and H. Patin, Chem. Rev., 2002, 102, 3757-377

[8] a) D. X. Li, Q. He, Y. Cui and J. B. Li, Chem. Mater., 2007, 19, 412Sun, S. J. Dong and E. K. Wang, Polymer, 2004, 45, 2181-2184; Q. Wang, G. J. Exarhos and A. D. Q. Li, J. Am. Chem. Soc., 2004, 120,

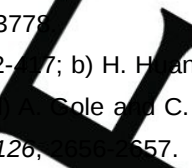

ang and X. (ng, Biomacromolecules, 2004, 5, 2340-2346; c) X. P. Several successful strategies have been investigated for making current ca. ods compatible with aqueous media. Notably, for a recent review on the use of environmentally friendly amphiphiles, see B. H. Lipshutz, N. A ey, J. C. Fennewald and E. D. Slack, Angew. Chem. Int. Ed., 2013, 52, 10952-10958.

[10] P. Zhao, N. Li, L. Salmon, N. Liu, J. Ruiz and D. Astruc, Chem. Commun., 2013, 3218-3220.

[11] E. Oh, K. Susumu, R. Goswami and H. Mattoussi, Lan 2010, 26. 7604-7f

[12] C. Gentilini, F. Evangelista, P. Rudolf, P. Franchi, M. Lucan Pasquate Am. Chem. Soc., 2008, 130, $15678-15682$.

[13] a) P. Pengo, S. Polizzi, M. Battagliarin, L. Pasquato and P. So and M. G. Ancona, Chem. Mater., 2002, 14, 2491-2408; c) W. P. Wut S. M. Gross, D. T. Miles and R. W. Murray, J. Am. Chem. Soc., 1998, 120, 12696--12697; d) H. Itsuka, Y. Akiyama, Y. N and P. V. Braun, Langmuir, 2004, 20,5613-5620, Tham and M. E. Williams, Langmuir, 2006, 22, 4319-4326; g) Y. Cheng, A. C. Samia, J. D. Meyers, I. Panagopoulos, B. Fei an Burda, J. Am. 2008, 130, 10643-10647; h) C. Gentilini, F. Evangelista, P. Rudolf, P. Franchi, M. Lucarini and L. Pasquato, J. Am. Chem. c., 2008, 130, 156 5682; i) L. Maus, J. P. Spatz and R. Fiammengo, Langmuir, $2009,25,7910-7917$.

[14] The formation of surface Au thiolate has b p confirmed by several authors, see for instance: a) P. D. Jadzinsky, G. Calero, C. J. Ackerson, D. A. Bushnell, R. D. Kornberg, Science, 2007, 318, 30-433; b) Y. Li, O. Zaluzhna, B. Xu, Y. Gao, J. M. Modest and Y. J. Tong, J. Am. Chem. Soc., 2011, 133, 2092-2095.

[15] a) A. Serra-Muns, R. Soler, E. M. Moreno-Mañas, R. Pleixats, R. M. Sebastián and A. Vallribera, New J. Chem., 2006, 30, 1584-1594; b) N. Mejías, A. Serra uns, R. Pleixats, A. Shafir and M. Tristany, Dalton Trans, 2009, 7748-7755; N. Mejías, R. Pleixats, A. Shafir, M. Medio-Simón and GAsensio, Eur. J. g. Chem., 2010, 5090-5099.

[16] W. Guo, R. Pleixat

[17] N. Li, P. Zhao, N. Lur, S. Salmon, J. Ruiz and D. Astruc, Chem. Eur. J., 2014, 20, 8363-8369. 
[18] a) S. Deki, K. Sayo, T. Fujita, A. Yamada and S. Hayashi, J. Mater. Chem., 1999, 9, 943-947; b) M. Iwamoto, K. Kuroda, V. Zaporojtchenko, S. Hayashi and F. Faupel, Eur. Phys. J. D, 2003, 24, 365-367; c) Y. M. A. Yamada, T. Arakawa, H. Hocke and Y. Uozumi, Angew. Chem. Int. Ed., 2007, 46, 704-706; d) E. Boisselier, A. K. Diallo, L. Salmon, J. Ruiz, and D. Astruc, Chem. Commun., 2008, 4819-4821; e) C. Ornellas, A. K. Diallo, J. Ruiz and D. Astruc, Adv. Synth. Catal., 2009, 351, 2147-2154; f) E. Boisselier, A. K. Diallo, L. Salmon, C. Ornelas, J. Ruiz and D. Astruc, J. Am. Chem. Soc., 2010, 132, 2729-2742.

[19] a) R. J. Chimentao, F. Medina, J. E. Sueiras, J. L. García Fierro, Y. Cesteros and P. Salagre, J. Mater. Sci., 2007, 42, 3307-3314; b) R. J. Chimentao, I. Kirm, F. Medina, X. Rodriguez, Y. Cesteros, P. Salagre and J. E. Sueiras, Chem. Commun., 2004, 846-847.

[20] a) B. L. Sanchez-Gaytan, Z. Qian, S. P. Hastings, M. L. Reca, Z. Fakhraai and S.-J. Park, J. Phys. Chem. C, 2013, 117, 8916- ’23; b) Y. Qiao, H. Chen, Y. Lin and J. Huang, Langmuir, 2011, 27, 11090-11097.

[21] PEG diffraction peaks consistent with the ICDD card 00-049-2095.

[22] FCC Au diffraction patterns consistent with the ICDD card 04-001-2616.

[23] K. Layek, M. Lakshmi Kantam, M. Shirai, D. Nishio-Hamane, T. Sasaki and H. Maheswaran, Green Chem., 2012, 14, 316

[24] M-L. Wang, T-T. Jiang, Y. Lu, H-J. Liu and Y. Chen, J. Mater. Chem. A, 2013, 1, 5923-5933.

[25] For some recent examples with gold nanoparticles and sodium borohydride, see: a) I. Biondi, G. Laurenczy and P. J. D. 8038-8045; b) P. Veerakumar, M. Velayudham, K.-L. Lu and S. Rajagopal, Appl. Catal. A Gen., 2012, 439-440, Chen, F. Cheng and S.-C. Jiang, J. Mater. Chem., 2012, 22, 21173-21182; d) M. M. Nigra, J-M. Ha and A. Katz 2983; e) D. Shah and H. Kaur, J. Molec. Catal. A Chem., 2014, 381, 70-76; f) S. Gatard, L. Salmon, C. Deraed Eur. J. Inorg. Chem., 2014, 2671-2677; g) R. Ciganda, N. Li, C. Deraedt, S. Gatard, P. Zhao, L. Salmon, R. Her Commun., 2014, 50, 10126-10129; h) S. Fountoulaki, V. Daikopoulou, P. L. Gkizis, I. Tamiolakis, G. S. Arm tas a 3504-3511; For $\mathrm{H}_{2}$ as reducing agent see, for instance: i) A. Corma and P. Serna, Nature Protocols, 2007, 17.

[26] TEM images of Au nanoparticles recovered after the last cycle showed nanoparticles aggregation and a partial loss of the stabilizer; see Supporting Information

[27] T. R. Jensen, M. L. Duval, K. L. Kelly, A. A. Lazarides, G. C. Schatz and R. P. Van Duyne, J. Phys. Chem. B, 1mand

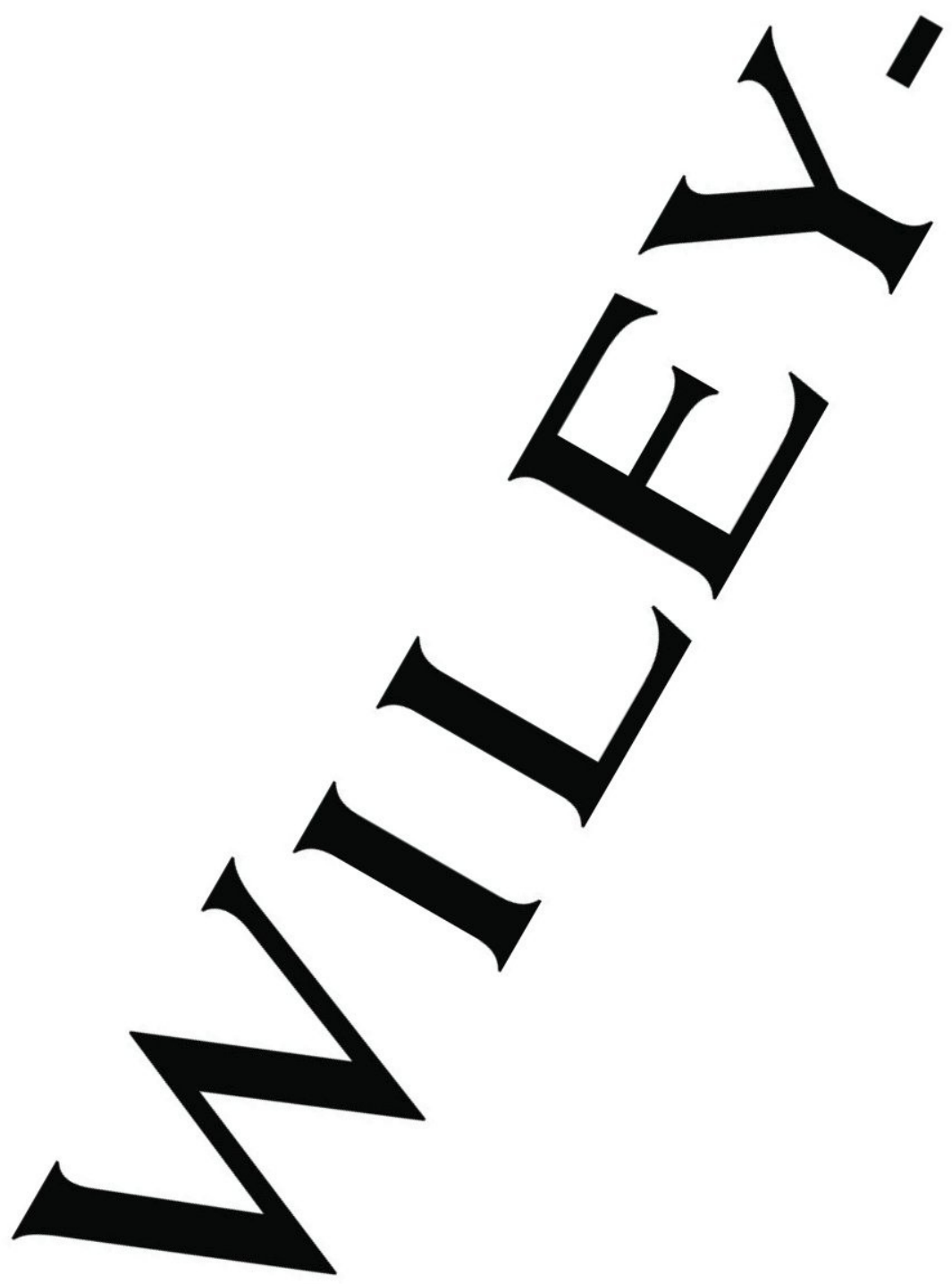


Water-soluble gold

nanoparticles were

synthesized using a PEG-

tagged N-rich stabilizer, $\mathbf{L}$.

The particle size can be finetuned via the $\mathbf{L} / \mathrm{Au}$ ratio. The new Au NPs are highly efficient and reusable in the selective reduction of $\mathrm{ArNO}_{2}$ in water. The particles also act as refractive index sensors via the shift in the UV absorption.

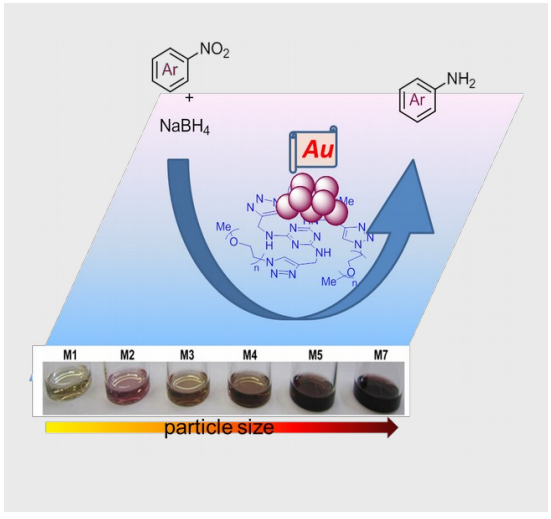

Wusheng Guo, Roser Pleixats* and Alexandr Shafir*

Page No. - Page No. Water-soluble Au nanoparticles: from catalytic selective nitroarene reduction in water to refractive index sensing
((Insert TOC Graphic here: max. width: $5.5 \mathrm{~cm}$; max. height: $5.0 \mathrm{~cm}$ )) 